\title{
Multifocusing imaging over an irregular topography
}

\author{
Boris Gurevich*, Shemer Keydar ${ }^{\ddagger}$, and Evgeny Landa
}

ABSTRACT
If seismic data are acquired over an irregular
topography, standard elevation statics methods may be
inaccurate because the assumption of vertical raypaths
will no longer be valid. An effective solution to the prob-
lem of irregular topography can be found through the use
of the multifocusing method, in which large supergath-
ers of seismic traces are stacked, each of which can span
many common midpoint (CMP) gathers. This can be
done by extending the multifocusing moveout formula
to explicitly account for nonzero elevations of the source
and receiver, as well as their horizontal coordinates.
Implementation of this formula into the multifocusing
algorithm is straightforward because estimating the nec-
essary raypath information (i.e., emergence angles) is an
integral part of the algorithm. The extended multifocus-
ing moveout correction can be applied directly to the
data acquired in areas of irregular topography without
the need for prior elevation static corrections. Synthetic
tests on such data show that the proposed technique
results in a better alignment of reflection events.

\section{INTRODUCTION}

Many seismic processing algorithms assume the data were recorded on a flat surface. When data are acquired over an irregular topography, elevation static corrections are often used to bring the data to a flat datum plane before NMO correction. However, raypaths are assumed to be vertical in static processes; the elevation corrections are imprecise and may introduce significant errors for large source-receiver offsets in areas with complex structure and large elevation variations.

Various approaches have been made to this problem, including wave equation datuming (Berryhill, 1984; Shtivelman and Canning 1984; Fowler and Schroeder, 2000) and prestack migration directly from irregular surfaces (Wiggins, 1984; Reshef, 1991; Beasley and Lynn, 1992). However, these methods are often computationally expensive and require prior knowledge of the velocity-depth model.

Thus, a simple ray-based procedure that could be used in the context of $\mathrm{NMO} /$ dip moveout (DMO)/stacking would be desirable. However, if the effects of nonvertical raypaths are significant, the time shifts caused by the elevation effect are no longer the same for the whole trace (static), but they are dependent on the emergence angles of the rays for individual reflections. Since raypaths are unknown beforehand, the correction for the elevation effect cannot be performed before, and independently of, any other processing. Dai and Cheadle (1995) propose a simple extension of the standard NMO equation to account for arbitrary source-receiver elevations. But since dip/emergence angle information is unavailable during the conventional NMO process, this procedure requires a special angle estimation algorithm.

An appealing alternative solution to the irregular topography problem can be obtained through the use of a multifocusing method (Gelchinsky et al., 1999a,b; Landa et al., 1999). The multifocusing method proposed by Gelchinsky et al. (1999a, b) belongs to a group of methods that can be characterized as macromodel-independent imaging methods. These methods, which also include the common-reflection-surface method (Mann et al., 1999; Jäger et al., 2001) and optical stack (de Bazelaire, 1988), are described in a collection of papers edited by Hubral (1999). Macromodel-independent imaging methods represent a new alternative to the classical processing sequence of NMO, DMO, and stacking. They represent a new transformation of 2-D multicoverage prestack data into a simulated zero-offset stacked section. This transformation involves stacking large supergathers of seismic traces, each of which can span many CMP gathers. Stacking is made possible by using a generalized moveout correction. For a given source-receiver pair this correction depends on three parameters: wave-front curvatures of the normal wave and the normal-incidence-point (NIP) wave (Hubral, 1983) and the emergence angle of the central ray. For each supergather and each zero-offset time $T_{0}$, these parameters are obtained through a coherence analysis of the moveout-corrected supergather. Because estimation of

Manuscript received by the Editor September 12, 2000; revised manuscript received May 14, 2001.

*Formerly Geophysical Institute of Israel; presently Curtin University of Technology, Department of Exploration Geophysics, GPO Box U1987, Perth, Western Australia 6845. E-mail: gurevich@geophy.curtin.edu.au.

†Geophysical Institute of Israel, P.O. Box 182, Lod 71100, Israel. E-mail: shemer@gii.co.il; evgeny@gii.co.il.

(C) 2002 Society of Exploration Geophysicists. All rights reserved. 
the emergence angle is an integral part of the multifocusing algorithm, its extension to areas of irregular topography is straightforward and can be achieved without additional cost.

\section{MULTIFOCUSING MOVEOUT FOR A FLAT ACQUISITION SURFACE}

Let us first consider acquisition on a flat surface (Figure 1). The central ray starts at $C$ (the central point) with an angle $\beta$ to the downward vertical (measured clockwise). It hits reflector $\Sigma$ at the normal-incidence point $N$ and returns to $C$. A paraxial ray from the source $S$ hits $\Sigma$ at $R$, intersects the central ray at $P$, and arrives back at the surface at the receiver location $G$. These two rays define a fictitious focusing wave which initially has the wavefront $\Sigma_{S}$, is reflected by the reflector $\Sigma$, focuses at $P$, and emerges again at $C$ with the wavefront $\Sigma_{G}$. The traveltime difference between the paraxial ray $S R G$ and the central ray $C N C$ (the so-called multifocusing moveout) can be written (Gelchinsky et al., 1999a; Landa et al., 1999)

$$
\Delta \tau=\Delta \tau^{+}+\Delta \tau^{-},
$$

where

$$
\Delta \tau^{+}=\frac{\sqrt{\left(R^{+}\right)^{2}+2 R^{+} X_{S} \sin \beta+X_{S}^{2}}-R^{+}}{V_{0}}
$$

is the traveltime difference between the $S R P$ section of the paraxial ray and the corresponding $C N P$ section of the central ray. The equation

$$
\Delta \tau^{-}=\frac{\sqrt{\left(R^{-}\right)^{2}+2 R^{-} X_{G} \sin \beta+X_{G}^{2}}-R^{-}}{V_{0}}
$$

solves the traveltime difference between the $P G$ section of the paraxial ray and the corresponding $P C$ section of the central ray. Here,

$$
\begin{aligned}
R^{+} & =\frac{1+\sigma}{\frac{1}{R_{N}}+\frac{\sigma}{R_{N I P}}}, \\
R^{-} & =\frac{1-\sigma}{\frac{1}{R_{N}}-\frac{\sigma}{R_{N I P}}},
\end{aligned}
$$

and $\sigma$ is the so-called focusing parameter, given by

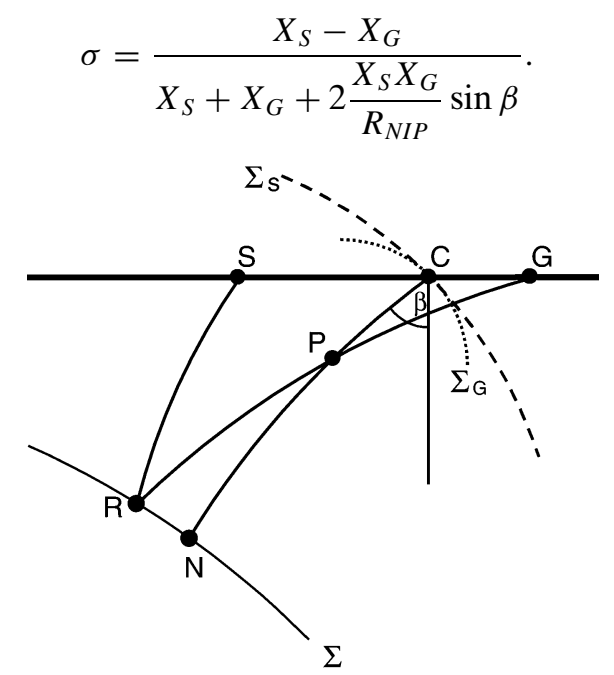

FIG. 1. Ray diagram of multifocusing moveout correction for a flat surface.
In equations (2)-(6), $X_{S}$ and $X_{G}$ are the source and receiver offsets for a given ray with respect to the central ray; $R^{+}$and $R^{-}$are the radii of curvature of the fictitious wavefronts $\Sigma_{S}$ and $\Sigma_{G}$ in the vertical plane, respectively; and $V_{0}$ is the near-surface velocity, which is assumed to be constant along the surface. Finally, $R_{N}$ and $R_{N I P}$ denote the radii of curvature of the two fundamental wavefronts, corresponding to the normal $(N)$ wave and normal-incidence-point (NIP) wave, respectively (Hubral, 1983). The wavefront of the normal wave is formed by normal rays emitted by different points on the reflector (as in an exploding reflector scenario). The NIP wavefront is formed by a point source placed at the point where the zero-offset ray emitted from the central point hits the reflector. The double square root in equation (1) can be understood using the concept of an auxiliary medium (Perroud et al., 1999; Cruz et al., 2000), which can be defined as a homogeneous medium whose velocity equals the near-surface velocity $V_{0}$. In the auxiliary medium both the central and paraxial rays are represented by combinations of straight-line segments.

Quantities $R^{+}$and $R^{-}$are radii of curvature of the fictitious wavefronts $\Sigma_{S}$ and $\Sigma_{G}$, respectively. It is clear from Figure 1 that, for a given central ray, the radii $R^{+}$and $R^{-}$depend upon the position of point $P$ where the paraxial ray intersects the central ray and thus upon the position of the source and receiver that define the paraxial ray. Equations (4) and (5) give the radii of curvature of these fictitious wavefronts $R^{+}$and $R^{-}$ in terms of the fundamental radii of curvature $R_{N}$ and $R_{N I P}$, which are defined by the central ray only and are the same for all source-receiver pairs in the vicinity of the central ray. The dependence of the radii $R^{+}$and $R^{-}$on the position of the source and receiver (or on the position of $P$ on the central ray) is contained in the focusing parameter $\sigma$, which has a very clear physical interpretation. In particular, $\sigma=0$ means that $R^{+}=R^{-}=R_{N}$, which implies that $P$ coincides with the center of curvature of the normal wavefront (or of the reflector) and corresponds to the case of coinciding source and receiver (zero-offset configuration). The case $\sigma=1(\sigma=-1) \mathrm{im}$ plies $R^{-}=0\left(R^{+}=0\right)$ and corresponds to the situation where the central point coincides with the source (receiver) location. When $\sigma=\infty$, then $R^{+}=R^{-}=R_{N I P}$ - the situation where $P$ coincides with $N$.

\section{EXTENSION TO AN ACQUISITION SURFACE WITH TOPOGRAPHIC RELIEF}

To apply the multifocusing method to seismic data acquired on an irregular surface, equations for the multifocusing moveout correction need to be extended to the situations where the source, receiver, and central point have different vertical coordinates. The derivations of the corresponding equations for the moveout correction and the focusing parameter are given below. For better clarity, the derivation is performed for a flat dipping reflector under a homogeneous overburden (Figure 2). For an inhomogeneous overburden this corresponds to an experiment in the auxiliary medium.

\section{Moveout correction}

The extension of equations (2) and (3) to an irregular topography is straightforward. Let $x_{0}, y_{0}$ be the horizontal and vertical coordinates of the central point $C$ and let $x_{S}, y_{S}$ and $x_{G}, y_{G}$ be the coordinates of the source $S$ and the receiver $G$, respectively, with rightward and upward positive directions for 
the $x$ - and $y$-axes. The $x$-coordinate of the point of intersection of the reflector and the datum $y=y_{0}$ is assumed to be zero. Let $Y_{G}=y_{G}-y_{0}$ be the receiver elevation from the datum, $X_{G}=x_{G}-x_{0}$ the receiver offset from the central trace, and $l=\sqrt{Y_{G}^{2}+X_{G}^{2}}$. From the triangle $C P G$ we get

$$
\begin{aligned}
\Delta \tau^{-} & =\frac{1}{v_{0}}[P G-P C] \\
& =\frac{1}{v_{0}}\left[\sqrt{\left(R^{-}\right)^{2}+l^{2}+2 R^{-} l \sin (\beta+\phi)}-R^{-}\right],
\end{aligned}
$$

where $\phi$ is the angle between the $C G$ vector and the positive $x$-axis, such that it is positive for positive $X_{G}$ and $Y_{G}$. We write

$$
\begin{aligned}
\sin (\beta+\phi) & =\sin \beta \cos \phi+\cos \beta \sin \phi \\
& =\frac{X_{G}}{l} \sin \beta+\frac{Y_{G}}{l} \cos \beta .
\end{aligned}
$$

Thus,

$$
\begin{aligned}
& \Delta \tau^{-}=\frac{1}{v_{0}} \\
& \times\left[\sqrt{\left(R^{-}\right)^{2}+\left(Y_{G}^{2}+X_{G}^{2}\right)+2\left(X_{G} \sin \beta+Y_{G} \cos \beta\right) R^{-}}-R^{-}\right] .
\end{aligned}
$$

Similarly,

$$
\begin{aligned}
& \Delta \tau^{+}=\frac{1}{v_{0}} \\
& \times\left[\sqrt{\left(R^{+}\right)^{2}+\left(Y_{S}^{2}+X_{S}^{2}\right)+2\left(X_{S} \sin \beta+Y_{S} \cos \beta\right) R^{+}}-R^{+}\right] .
\end{aligned}
$$

where $R_{N I P}=C N$ and $R^{-}=P C(P$ is the focusing point $)$. Solving for $\sigma$ yields

$$
\sigma=-\frac{R_{N I P}}{R^{-}-R_{N I P}} .
$$

To find $\sigma$, we need to determine the coordinates of $P$, which is the point of intersection of the normal ray $C N$ and the reflected ray $R G$, originating from the imaginary source $S^{*}$.

The location of $S^{*}$ is defined by the location of the source $S\left(x_{s}, y_{s}\right)$ and the reflector $\Sigma$, the coordinates of which are given by the equation

$$
y_{\Sigma}(x)=y_{0}-\left(x-x_{0}\right) \tan \beta-\frac{R_{N I P}}{\cos \beta} .
$$

To find the coordinates $x_{1}, y_{1}$ of the imaginary source $S^{*}$, we first calculate the distance $d=A S$ from the source $S$ to the reflector:

$$
\begin{aligned}
d=\left(y_{S}-y_{\Sigma}\left(x_{S}\right)\right) \cos \beta= & \left(y_{S}-y_{0}\right) \cos \beta \\
& +\left(x_{S}-x_{0}\right) \sin \beta+R_{N I P} .
\end{aligned}
$$

The coordinates $x_{1}, y_{1}$ can then be written as

$$
\begin{aligned}
& x_{1}=x_{S}-2 d \sin \beta, \\
& y_{1}=y_{S}-2 d \cos \beta .
\end{aligned}
$$

Point $P$ is defined as the intersection point of the ray $S^{*} G$,

$$
\frac{x-x_{G}}{x_{1}-x_{G}}=\frac{y-y_{G}}{y_{1}-y_{G}},
$$

with the normal ray $C N$ passing through the central point,

$$
y=y_{0}+\left(x-x_{0}\right) \cot \beta .
$$

Solving the system of equations (10) and (11) for $x$ and $y$ yields

$$
\begin{aligned}
& x_{p}=-\frac{y_{0} x_{1}-y_{0} x_{G}-x_{0}(\cot \beta) x_{1}+x_{0}(\cot \beta) x_{G}+x_{G} y_{1}-y_{G} x_{1}}{-y_{1}+y_{G}+(\cot \beta) x_{1}-(\cot \beta) x_{G}}, \\
& y_{p}=-\frac{(\cot \beta) x_{G} y_{1}-(\cot \beta) y_{G} x_{1}+y_{0} y_{1}-y_{0} y_{G}-x_{0}(\cot \beta) y_{1}+x_{0}(\cot \beta) y_{G}}{-y_{1}+y_{G}+(\cot \beta) x_{1}-(\cot \beta) x_{G}} .
\end{aligned}
$$

\section{Focusing parameter}

In general, the focusing parameter is a means of obtaining $R^{+}$and $R^{-}$. Conversely, if we know $R^{+}$and $R^{-}$, we can obtain $\sigma$ from equations (4) and (5). For a flat dipping reflector the normal-wave radius $R_{N}$ is infinite. Thus, $\sigma$ can be obtained from just one of these equations. In particular, equation (5) for a flat reflector $\left(R_{N}=\infty\right)$ has the form

$$
R^{-}=\frac{\sigma-1}{\sigma} R_{N I P}
$$

The radius $R^{-}$can be found as the distance $P C$ between the intersection point $P$ and the central point $C$ :

$$
\begin{aligned}
R^{-} & =\frac{y_{0}-y_{p}}{\cos \beta} \\
& =\frac{y_{0} x_{1}-y_{0} x_{G}+x_{G} y_{1}-y_{G} x_{1}-x_{0} y_{1}+x_{0} y_{G}}{-y_{1} \sin \beta+y_{G} \sin \beta+(\cos \beta) x_{1}-(\cos \beta) x_{G}} .
\end{aligned}
$$

Finally, substituting $R^{-}$into equation (9) yields

$$
\sigma=\frac{X_{S}-X_{G}-\left(Y_{S}-Y_{G}\right) \tan \beta}{X_{S}+X_{G}-\left(Y_{S}+Y_{G}\right) \tan \beta+\frac{2\left(X_{S} X_{G}-Y_{S} Y_{G}\right)+\left(X_{S} Y_{G}+X_{G} Y_{S}\right)\left(\cos ^{2} \beta-\sin ^{2} \beta\right)}{R_{N I P}} \sin \beta} .
$$


Note that for a flat datum $Y_{S}=Y_{G}=0$, equation (13) reduces to equation (6).

\section{IMPLEMENTATION}

To use multifocusing stacking for a flat topography for each $T_{0}$ on each zero-offset trace, it is necessary to determine three imaging parameters: $\beta, R_{N I P}$, and $R_{N}$. In the conventional NMO stack, only a single parameter, the stacking velocity, must be determined. Implementation of the multifocusing method is based on a coherence analysis of the signal on the observed seismic traces. The data are moveout corrected along different traveltime curves according to equations (1)-(6) to find the curve closest to the traveltime curve for the signal. The unknown parameters $\beta, R_{N I P}$, and $R_{N}$ are estimated by finding a set of parameters that maximizes the semblance function. Semblance is calculated over the supergather in a specified time window along trial traveltime curves. Maximization of the semblance is achieved by a nonlinear optimization method. This correlation procedure is repeated for each central point and for each time sample, forming a multifocusing time section. Each sample on this section represents the optimal stacked value corresponding to the optimal values of the moveout parameters $\beta, R_{N I P}$, and $R_{N}$. Multifocusing parameter estimation requires global maximization of the semblance as a function of the three multifocusing parameters. In the presence of noise, this function may have many maxima; thus, the search for a global maximum poses a global optimization problem. A systematic approach to this problem that utilizes unique properties of the objective function has been proposed by Jäger et al. (2001).

The multifocusing stacking of data acquired in areas with an irregular topography is performed in exactly the same way as for a flat topography, except that equations (7), (8), and (13) are used in place of equations (2), (3), and (6). Since the topography is assumed to be known, multifocusing parameter estimation requires searching for the same triplet of parameters- $\beta$, $R_{N I P}$, and $R_{N}$-as in the case of a flat surface. One parameter

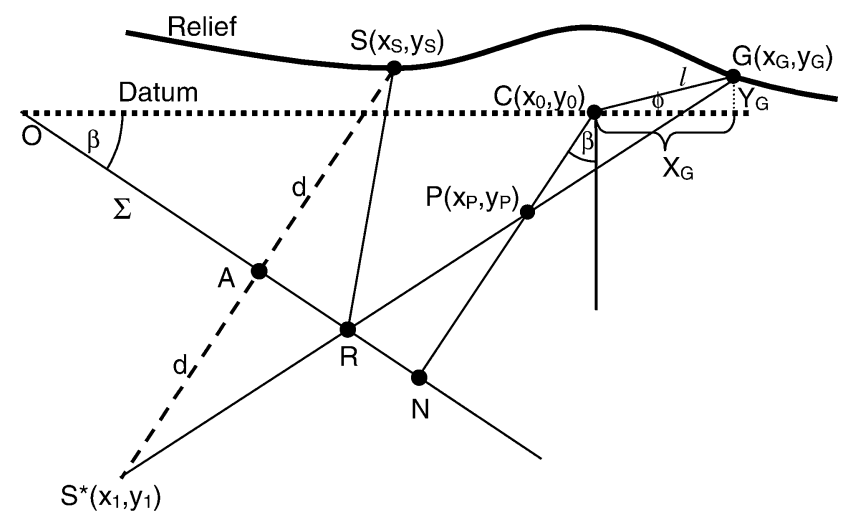

FIG. 2. Ray diagram of multifocusing moveout from topography.

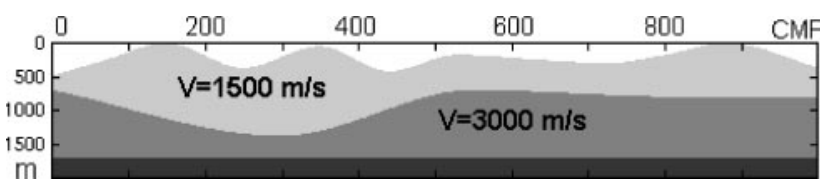

FIG. 3. Velocity-depth model with topographic variation. that must be fixed is the elevation of the central point $y_{0}$. To obtain a stacked section for a flat datum, this parameter should be set the same for all central traces, i.e., $y_{0}=$ constant. However, one can also choose to construct a stacked section from the actual acquisition surface, or from a floating datum, by choosing an appropriate datum elevation curve $y_{0}\left(x_{0}\right)$. Practical experience has shown that multifocusing parameter estimation is more stable when the elevation of the central point is closer to the actual acquisition surface. From this perspective, the use of a floating datum is probably the most practical.

\section{EXAMPLES}

To illustrate how the multifocusing algorithm could handle data from an area of irregular topography, a synthetic wavefield was developed. The model was a simple three-layer case (Figure 3) with an irregular topography, and it was computed

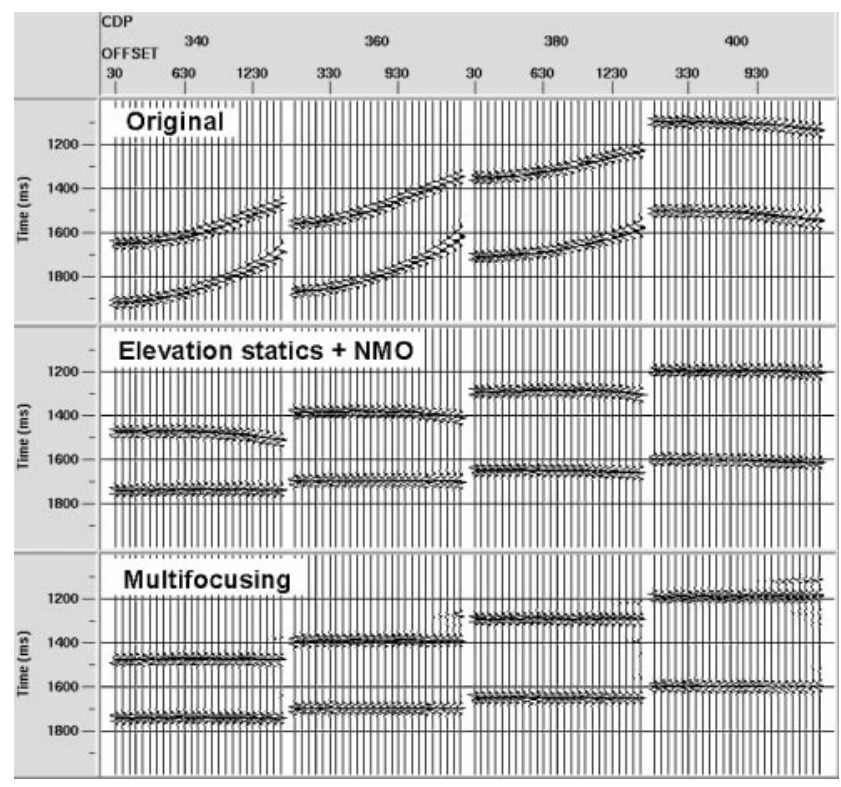

FIG. 4. Synthetic CMP gathers for the model shown in Figure 3. (Top) Original gathers. (Middle) Gathers after elevation statics and NMO correction. (Bottom) Gathers after multifocusing moveout correction.

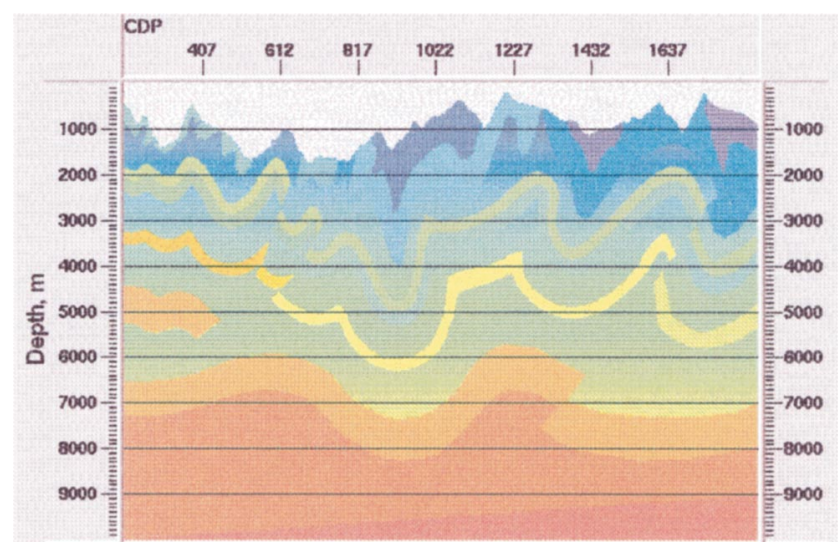

FIG. 5. Velocity-depth model with rough topography simulating a foothills environment. Velocities vary from $2000 \mathrm{~m} / \mathrm{s}$ (blue) to $6000 \mathrm{~ms} / \mathrm{s}$ (red). 


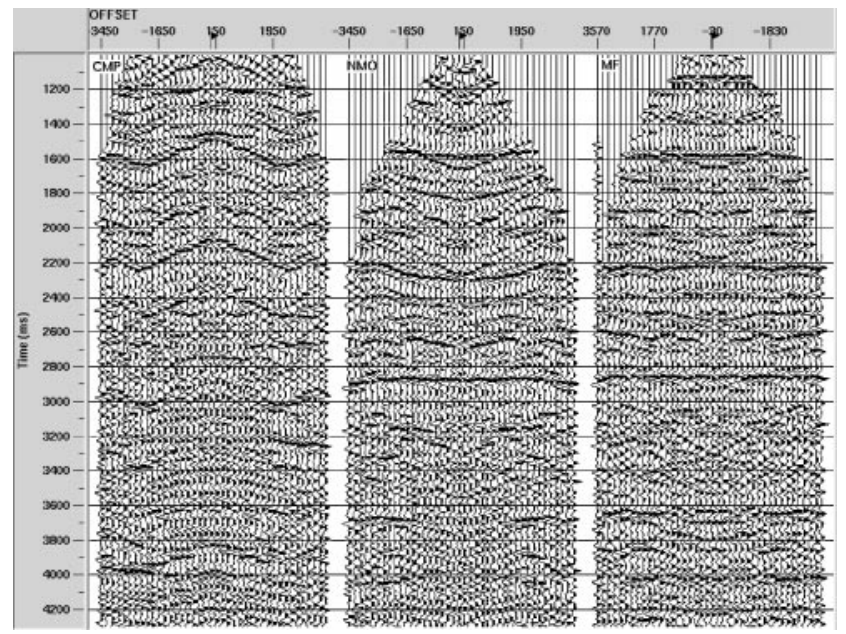

FIG. 6. Synthetic CMP gather for the model shown in Figure 5. (Left) Original gather. (Center) After elevation statics and NMO correction. (Right) After multifocusing moveout correction.

using a simple ray-tracing algorithm. The data were processed using two processing flows. In the first flow, the data were moved to a flat datum by applying elevation statics. The resulting flat-datum data were subjected to the standard velocity analysis followed by NMO correction. In the second flow, the data were analyzed with the automatic multifocusing parameter estimation extended to handle irregular topography. The process used supergathers each containing 15 common midpoint (CMP) gathers. Then, the multifocusing moveout correction with the estimated multifocusing parameters was applied to the original prestack data. Figure 4 shows three CMP gathers. The original CMP gathers are shown at the top, the NMO-corrected gathers are in the middle, and the gathers after multifocusing are at the bottom. It is clear that the multifocusing moveout correction aligns the reflections at large offsets more satisfactorily than the conventional approach based on elevation statics and NMO correction.

For a more realistic example, a synthetic data set was computed using a finite-difference method for a complex model with a rough topography simulating a foothills environment (Figure 5). Figure 6 shows one of the CMP gathers from this data set. As a result of the rough topography, the reflection moveouts in the original CMP gather (left) are quite complicated. The central panel shows the same gather after applying elevation statics, velocity analysis, and NMO correction. The right panel shows the same gather after automatic multifocusing parameter estimation, and application of the multifocusing moveout correction. Again, we see that the multifocusing method improves the alignment of the reflected events.

\section{CONCLUSIONS}

The multifocusing method can be extended to account for the irregular topography of the acquisition surface. This is done by extending the multifocusing moveout correction formula for an irregular acquisition surface. The extended multifocusing moveout correction depends explicitly on the elevations of the source and receiver as well as their horizontal coordinates. Implementation of this formula into the multifocusing algorithm is straightforward because estimation of the necessary raypath information (i.e., emergence angles) is an integral part of the algorithm.

Extended multifocusing moveout corrections can be applied directly to the data acquired in areas with irregular topography without the need for prior elevation static corrections. Our results show a better alignment of reflection events in two examples where the assumption of vertical raypaths, on which the elevation statics approach is based, is no longer valid.

\section{ACKNOWLEDGMENTS}

The research was carried out with the support of the Israel Ministry of National Infrastructures. We are grateful to Igor Belfer and Pinchas Trachtman for assistance with modeling and processing the data. We also thank Moshe Reshef for useful discussions.

\section{REFERENCES}

Beasley, C., and Lynn, W., 1992, The zero-velocity layer: Migration from irregular surfaces: Geophysics, 57, 1435-1443.

Berryhill, J. R., 1984, Wave equation datuming before stack (short note): Geophysics, 49, 2064-2067.

Cruz, J. C. R., Hubral, P., Tygel, M., Schleicher, J., and Höcht, G., 2000, The common reflecting element (CRE) method revisited: Geophysics, 65, 979-993.

Dai, N., and Cheadle, S., 1995, Two-parameter NMO processes from surface: Ann. Mtg., Can. Soc. Expl. Geophys., Extended Abstracts, 135-136.

de Bazelaire, E., 1988, Normal moveout revisited-Iingomogeneous media and curved interfaces: Geophysics, 53, 143-157.

Fowler, P., and Schroeder, S., 2000, An accurate and efficient hybrid method for poststack topographic datuming: 70th Ann. Internat Mtg., Soc. Expl. Geophys., Expanded Abstracts, 2036-2039.

Gelchinsky, B., Berkovitch, A., and Keydar, S., 1999a, Multifocusing homeomorphic imaging, part 1-Basic concepts and formulae: J. Appl. Geophys., 42, 229-242.

1999b, Multifocusing homeomorphic imaging, part 2Multifold data set and multifocusing: J. Appl. Geophys., 42, 243-260 Hubral, P., 1983, Computing true amplitude reflections in a laterally inhomogeneous earth: Geophysics, 48, 1051-1062.

Ed., 1999, Macro-model independent seismic reflection imaging: J. Appl. Geoph., 42,137-348.

Jäger, R., Mann, J., Höcht, G., and Hubral, P., 2001, Commonreflecting-surface stack: Image and attributes: Geophysics, 66, 97109.

Landa, E., Gurevich, B., Keydar, S., and Trachtman, P., 1999, Application of multifocusing method for subsurface imaging: J. Appl. Geophys., 42, 283-300.

Mann, J., Jäger, R., Müller, T., Höcht, G., and Hubral, P., 1999 Common-reflecting-surface stack $-A$ real data example: J. Appl. Geophys., 42, 301-318.

Perroud, H., Hubral, P., and Höcht, G., 1999, Common-reflection-point stacking in laterally inhomogeneous media: Geophys. Prosp., 47, 124.

Reshef, M., 1991, Depth migration from irregular surfaces with depth extrapolation methods (short note): Geophysics, 56, 119-122.

Shtivelman, V., and Canning, A., 1984, Datum correction by waveequation extrapolation: Geophysics, 53, 1311-1322.

Wiggins, J. W., 1984, Kirchhoff integral extrapolation and migration of nonplanar data: Geophysics, 49, 1239-1248. 